\title{
Propriedade da Fibra de Coco: Uma Revisão Sistemática
}

\author{
Properties of Coconut Fiber: Systematic Review
}

\author{
Mariana Aparecida Giraldelli*a; Osvaldo Alves Pereira ${ }^{\mathrm{a}}$; Samuel Felipe dos Santos ${ }^{\mathrm{b}}$; Mirela Aguiar Brasil \\ Stefani Karoline Teodoro Pinheiro ${ }^{\mathrm{b}}$
}
${ }^{a}$ Universidade de Cuiabá, Programa de Pós-Graduação Stricto Sensu em Ciências Ambientais. MT, Brasil.
bUniversidade de Cuiabá. MT, Brasil.
*E-mail: marianagiraldelli5@gmail.com

\begin{abstract}
Resumo
O coco é um fruto presente na agricultura de diversos países, estimando-se um consumo 61,3 milhões de toneladas colhidas por ano em cerca de 12 milhões de hectares. O principal consumo do fruto está relacionado com a indústria alimentícia, sendo consumida a sua água, a polpa que é a parte comestível do fruto e que gera demais derivados alimentícios. Entretanto, após a utilização das partes comestíveis do fruto, é possível utilizar o seu subproduto extraindo a fibra do mesocarpo do fruto, sendo este compósito conhecido como fibra de coco. A fibra vegetal proveniente do coco, fibra de coco, é amplamente utilizada em diversos setores econômicos em função de suas características de durabilidade, rigidez, impermeabilidade, resistência contra ataques biológicos, resistência à tração e termoacústica, se destacando das demais fibras vegetais. Estas características estão ligadas com a sua composição de lignocelulósicos desta fibra, sendo que esta apresenta maiores concentrações de lignina (45 - 46\%) em comparação com outras fibras naturais. Esta revisão narrativa é baseada em livros, artigos, seminários e demais obras científicas que abordam o tema de fibras vegetais e fibra de coco, sendo que o principal objetivo é fazer um levantamento sobre este compósito abordando sua origem, comportamentos químicos, físicos, mecânicos e características gerais.
\end{abstract}

Palavras-chave: Fibra de Coco. Coco. Fibra Vegetal.

\begin{abstract}
Coconut is a fruit present in agriculture in several countries, with an estimated consumption of 61.3 million tons of tons per year in about 12 million hectares. The main fruit consumption is related to the food industry, being consumed its water, the pulp that is the edible part of the fruit and that generates other food derivatives. However, after using the edible parts of the fruit, it is possible to use its by-product by extracting the fiber from the fruit's mesocarp, this composite being known as coconut fiber. Vegetable fiber from coconut, coconut fiber, is widely used in several different sectors due to its characteristics of durability, rigidity, impermeability, resistance against biological resistance, tensile and thermoacoustic resistance, standing out from other vegetable fibers. These characteristics are associated with its lignocellulosic composition of this fiber, being present higher lignin (45-46\%) in comparison with other natural fibers. This narrative review is based on books, articles, seminars and other scientific works that address the topic of vegetable fibers and coconut fiber, and the main objective is to survey this composite addressing its origin, chemical, organic, mechanical and general features.
\end{abstract}

Keywords: Coconut Fiber. Coconut. Vegetable Fiber.

\section{Introdução}

O fruto coco (Cocos nucifera, Arecaceae) é proveniente da palmeira coqueiro (Cocos nucifera) e seu cultivo está presente nas atividades agrícolas de mais de 90 países, sendo empregado, principalmente, no ramo alimentício (BRAINER, 2018).

As fibras de coco são fibras vegetais oriundas de subprodutos da agricultura, sendo extraídas após a utilização principal do fruto. As fibras são obtidas da casca do fruto e podendo ser extraídas tanto do coco seco como verde. Conforme Bhatia e Smith (2008), a utilização desta fibra na indústria é vasta, estando presente em diversos setores como automotivo, geo-ambiental e construtivo. Passos (2005) afirmou que a utilização em demasia da fibra está relacionada com a sua composição química, física e mecânica que se difere das demais fibras vegetais e permite uma utilização de maneira mais ampla.
Este estudo foi realizado com o objetivo de conhecer melhor a fibra oriunda do coco, abordando a sua origem, morfologia do fruto, obtenção da fibra, características do compósito comparando-a com outras fibras de origem vegetal. Estudo é fundamentado em revisões bibliográficas, utilizando como base pesquisas de caráter acadêmico através de consultas em livros, publicações, artigos e revistas que abordam a temática.

\section{Desenvolvimento}

\subsection{Metodologia}

A revisão bibliográfica foi realizada por meio de consultas aos bancos de dados do portal de periódicos CAPES/ MEC, Scientific Electronic Library Online (SciELO), Google Scholar, Scopus (Elsevier), livros consagrados que abordassem o tema de biologia vegetal e fibras vegetais. O critério de inclusão ou exclusão dos artigos ocorreu a partir 
do título, resumo e das palavras-chaves, sendo utilizado as seguintes palavras-chaves para busca: fibra, coco verde, fibra de coco.

\subsection{Fibras vegetais}

A incorporação de fibras naturais na construção de habitações é ancestral e remota a antiga civilização egípcia e mesopotâmia. A utilização deste compósito está vinculada ao seu baixo custo e disponibilidade abundante, o que leva a diversas pesquisas sobre o comportamento destes materiais, suas adequações e possíveis substituições de fibras sintéticas por naturais nas tecnologias construtivos atuais (CABRERA; NWAUBANI, 1990).

As fibras naturais são subdivididas de acordo com sua origem, proveniente de vegetais, animais ou minerais. Conforme Pritchard, Sarsby e Anand (2000), as fibras de origem vegetais possuem maior potencial para serem utilizadas na engenharia em função de suas propriedades. As fibras de origem animal, por exemplo, possuem menor resistência e maior alongamento em comparação as fibras vegetais e as fibras mineiras possuem maior valor aquisitivo, sendo mais quebradiças, carecendo de força e flexibilidade.

As fibras vegetais são constituídas, basicamente, de celulose e podem ser classificadas morfologicamente, de acordo com a parcela da planta da qual é extraída, sendo divididas em fibras da semente, caule e folha. O diagrama apresentado, na Figura 01, expõe esta classificação das fibras naturais, nas quais é possível visualizar as subdivisões da fibra vegetal e os principais exemplos de cada compósito (BLEDZKI; SPERDER; FARUK, 2002).

Figura 1 - Diagrama das classificações de fibras naturais, expondo a subclassificação da fibra vegetal com exemplo dos principais compósitos

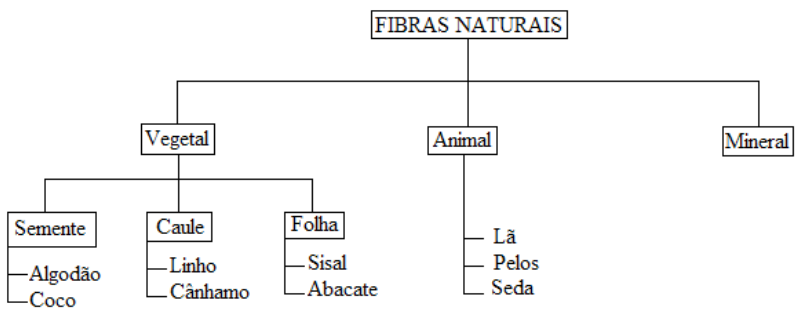

Fonte: Adaptado Bledzki, Sperder e Faruk (2002).

Ainda, no que se diz respeito às diferenças das fibras vegetais, as composições químicas e físicas podem variar amplamente (Quadro 1). Conforme Rowell, Han e Rowell (2000), tais variações ocorrem de planta em planta, também variando entre plantas de diferentes localizações geográficas, idades, climas e condições do solo.

Quadro 1 - Composição química e física de diversas fibras vegetais

\begin{tabular}{|c|c|c|c|c|c|}
\hline \multicolumn{7}{|c|}{ Fibras Vegetais: Composição química e física } \\
\hline \multirow{2}{*}{ Classificação } & Fibra & Celulose (\%) & Lignina (\%) & Comprimento (mm) & Largura (\%) \\
\hline \multirow{2}{*}{ Caule } & Cânhamo & $57,0-78,0$ & $3,7-13,0$ & $5,0-55,0$ & $0,01-0,04$ \\
\cline { 2 - 7 } & Linho & $29,0-81,0$ & $2,0-23,0$ & $4,0-77,0$ & $0,005-0,04$ \\
\hline \multirow{2}{*}{ Folha } & Abacate & $56,0-64,0$ & $7,0-12,0$ & $2,0-12,0$ & $0,02-0,03$ \\
\cline { 2 - 7 } & Sisal & $43,0-88,0$ & $4,0-12,0$ & $0,8-8,0$ & $0,008-0,04$ \\
\hline \multirow{2}{*}{ Semente } & Algodão & $80,0-99,0$ & $0,5-66,0$ & $10,0-60,0$ & $0,01-0,04$ \\
\cline { 2 - 7 } & Coco & $35,0-45,0$ & $45,0-46,0$ & $0,3-1,0$ & $0,10-0,45$ \\
\hline
\end{tabular}

Fonte: Adaptado Bhatia e Smith (2008).

As variações nas composições químicas e físicas das fibras levam a variações em suas propriedades mecânicas (Quadro 2). Bhatia e Smith (2008) defendem que mesmo com variações em sua resistência, as utilizações das fibras naturais na engenharia agregam valor ao produto final.

Quadro 2 - Propriedades mecânicas de diversas fibras vegetais

\begin{tabular}{|c|c|c|c|c|}
\hline \multicolumn{5}{|c|}{ Fibras Vegetais: Propriedades Mecânicas } \\
\hline Classificação & Fibra & $\begin{array}{l}\text { Resistência a } \\
\text { Tração (MPa) }\end{array}$ & $\begin{array}{c}\text { Modulo de Elasticidade } \\
\text { (GPa) }\end{array}$ & $\begin{array}{l}\text { Alongamento de } \\
\text { Ruptura (\%) }\end{array}$ \\
\hline \multirow{2}{*}{ Caule } & Cânhamo & $580,0-110,0$ & $3,0-90,0$ & $1,3-4,7$ \\
\hline & Linho & $343,0-100,0$ & $27,0-100,0$ & $1,6-3,2$ \\
\hline \multirow{2}{*}{ Folha } & Abacate & $980,0-000$ & 72,0 & $2,5-12,0$ \\
\hline & Sisal & $468,0-55,0$ & $9,0-28,0$ & $1,9-4,5$ \\
\hline \multirow{2}{*}{ Semente } & Algodão & $287,0-97,0$ & $5,5-12,6$ & $2,0-10,0$ \\
\hline & Coco & $106,0-70,0$ & $3,0-6,0$ & $15,0-47,0$ \\
\hline
\end{tabular}

Fonte: Adaptado Bhatia e Smith (2008)

No Brasil, as técnicas construtivas tradicionais comumente utilizam fibras vegetais e animais de fácil acesso como: o esterco, a palha, o feno, entre outros. Minke (2012) descreveu que a incorporação de fibras ao barro é realizada para controlar a retração durante a secagem, melhorando a força de ligação da mistura, reduzindo a densidade e diminuindo a aparência 
de rachadura nos blocos.

\subsection{Fibra de coco}

As fibras de coco são oriundas de subprodutos da agricultura, sendo geradas após a utilização dos produtos principais; água de coco e a parte comestível do fruto. Este compósito é obtido através da casca do coco, fruto proveniente da palmeira coqueiro (Cocos nucifera), sendo possível extração tanto no fruto maduro como verde (BARBOSA et al., 2016).

Passos (2005) destacou que a fibra de coco possui maiores concentrações de tanino e lignina do que as fibras vegetais convencionais, assim alterando o seu comportamento mecânico e habilitando-a a ser utilizada de maneira diversas. O Quadro 3 apresenta as características química, física e mecânicas da fibra de coco.

Quadro 3 - Características químicas, físicas e mecânicas da fibra de coco

\begin{tabular}{|c|c|c|}
\hline \multicolumn{3}{|c|}{ Características da Fibra de Coco } \\
\hline \multirow{3}{*}{$\begin{array}{c}\text { Composições } \\
\text { Química } \\
\text { Física }\end{array}$} & Celulose (\%) & $35,0-45,0$ \\
\cline { 2 - 3 } & Lignina (\%) & $45,0-46,0$ \\
\cline { 2 - 3 } & Comprimento (mm) & $0,3-1,0$ \\
\cline { 2 - 3 } & Largura (\%) & $0,10-0,45$ \\
\hline \multirow{2}{*}{$\begin{array}{c}\text { Propriedades } \\
\text { Mecânicas }\end{array}$} & Resistência a Tração (MPa) & $106,0-70,0$ \\
\cline { 2 - 3 } & Modelo de Elasticidade (GPa) & $3,0-6,0$ \\
\cline { 2 - 3 } & Alongamento de Ruptura (\%) & $15,0-47,0$ \\
\hline
\end{tabular}

Fonte: Adaptado Bhatia e Smith (2008).

O uso da fibra de coco na engenharia é vasto. A sua utilização está presente em materiais automotivos (como acolchoados e isolante), geoambiental (biomanta para estabilização de solo) e em materiais construtivos (enchimento de paredes, biomanta termo acústica) (BHATIA; SMITH, 2008).

A sua versatilidade também permite o seu uso em construções em terra crua, entretanto, a sua utilização nestas técnicas é pouco usual no Brasil. A baixa utilização ocorre pela dificuldade de extração deste compósito.

\subsubsection{Coqueiro e o fruto coco}

"O coqueiro é a palmeira de maior importância socioeconômica das regiões intertropicais do Mundo. Pela magnitude dos produtos obtidos das diferentes partes da planta, pode-se afirmar que do coqueiro tudo se aproveita" (ARAGÃO et al., 2002).

Raven, Evert e Eichhorn (2014) descrevem que o coqueiro (Cocos nucifera) (Figura 2), é uma planta cultivada nos trópicos, possivelmente, originado no Pacífico Ocidental ou em região tropical da Ásia. O fruto do coqueiro, coco (Cocos nucifera, Arecaceae) (Figura 3) é adaptado para dispersão por correntes oceânicas, o que permitiu a sua difusão pelo Pacífico Ocidental e central, antes das viagens europeias exploratórias. A existência abundante do coqueiro nas regiões tropicais pode ser decorrente da dispersão natural e não da intervenção humana.
Figura 2 - Ilustração de um coqueiro e do fruto

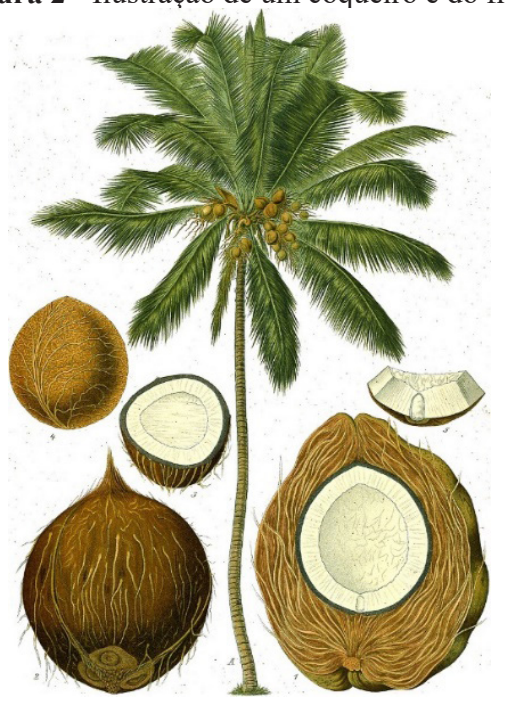

Fonte: Kohler - Repositório Digital da Wikipedia. (1987)

Figura 3 - Cacho de coco

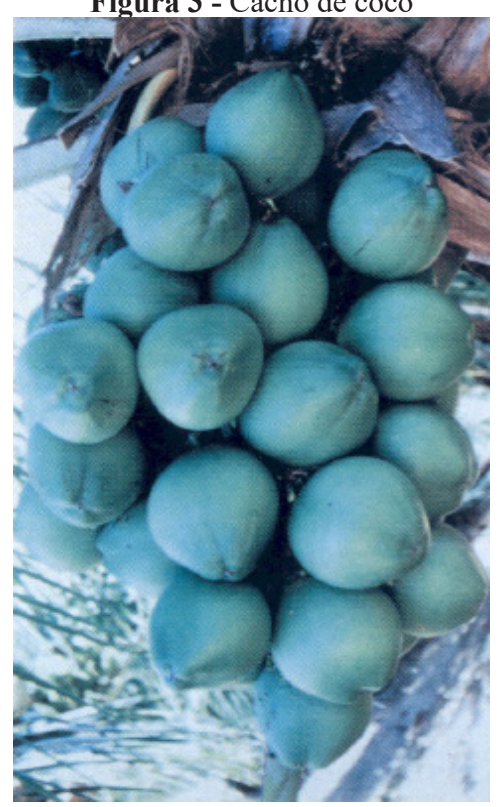

Fonte: Resende (2002).

O cultivo do coqueiro está presente nas atividades agrícolas em mais de 90 países, afetando diretamente a economia com empregos diretos e alimentação. Em 2018, a produção mundial do coco foi estimada em 61,3 milhões de toneladas com a área mundial colhida com cerca de 12 milhões de hectares (BRAINER, 2018).

Os maiores produtores mundiais de coco, conforme dados de 2018, são a Indonésia, seguida pelas Filipinas e a Índia que ocupam o terceiro lugar de produção. O Brasil é o sexto em maior área cultivada, sendo que o fruto é cultivado em todas as regiões do país (BRAINER, 2018).

Barbosa et al. (2016) destacaram que, anualmente, o Brasil produz cerca de 8,1 bilhões de unidades de coco que geram $70 \%$ do resíduo encontrado nas praias, tendo como destinação final lixões e aterros e, assim, diminuindo a vida útil desses, pois este resíduo apresenta alto volume, leva cerca 
de 8 anos para sua decomposição completa, sendo potenciais emissores de gases estufa, contaminam o solo e podem contribuir na proliferação de animais peçonhentos. Desse modo, a reutilização deste material se torna uma alternativa atrativa para o meio ambiente.

\subsubsection{Morfologia}

"O coqueiro pertence ao reino vegetal, ramo phanerogamos, sub-ramo angiospermas, classe monocotiledônea, ordem príncipes, família Arecaceae, espécie Cocos nucifera L." (ALVES; MULLER, 1995).

$\mathrm{O}$ fruto do coqueiro (Figura 4) consiste em quatro partes básicas:

- Epiderme lisa: superfície dura e brilhante que forma a casca e envolve o mesocarpo.

- Mesocarpo: parte espesso e fibroso, cujo principal interesse desta pesquisa, que envolve o endocarpo.

- Endocarpo: camada rígida que envolve a amêndoa do fruto.

- Amêndoa: parte comestível do fruto, sendo formada pelo o albúmen sólido (camada carnosa, branca e oleosa), o albúmen líquido (água) e o embrião.

Figura 4 - Ilustração de corte frontal do coco-da-baía

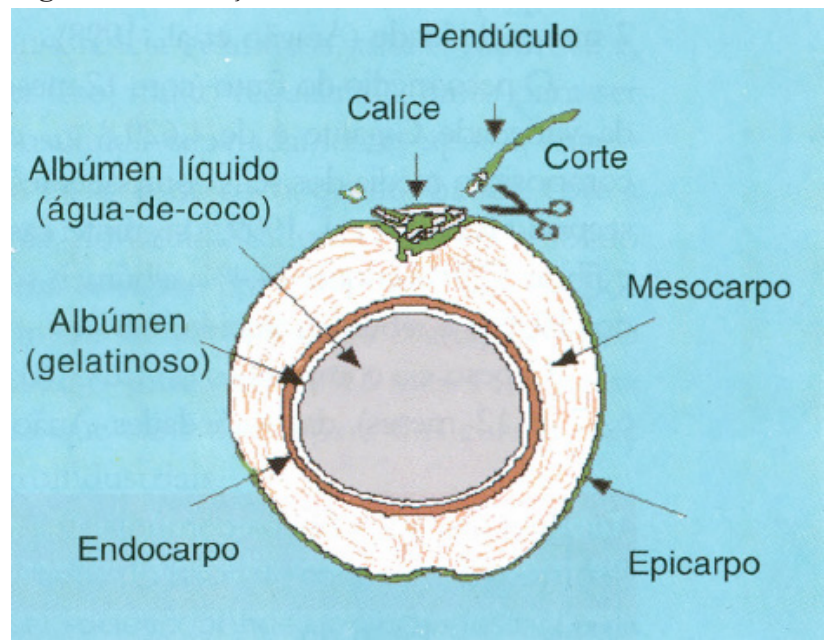

Fonte: Aragão et al. (2002).

Raven, Evert e Eichhorn (2014) afirmaram que o coco é um fruto rico nutricionalmente. O albumen sólido (carne do coco) é rico em gordura, carboidratos e moderada quantidade de proteína. O albumen líquido (água de coco) possui carboidratos, gordura, proteínas e diversos minerais.

Os albumens são os principais produtos do coco por serem utilizados para consumo humano. As cascas e a fibras do fruto, subprodutos, são utilizadas para a produção de muitos itens como tecidos, construções e utensílios.

\subsubsection{Obtenção das fibras}

As fibras do coco são obtidas do mesocarpo do fruto, que é formado por fibras e pela casca do fruto, também conhecido como "pó".

Conforme Rosa et al. (2001), a fibra e o pó do coco podem ser extraídos tanto do coco verde ou do coco seco, entretanto, as fibras extraídas do fruto verde não apresentam as mesmas características da fibra do coco maduro, apresentando alto teor de umidade, cerca de $85 \%$, o que pode desencorajar a utilização da fibra do coco verde.

\subsubsection{Vantagens da utilização}

A fibra de coco é um material versátil e que apresenta inúmeros métodos de utilização em função de sua resistência e durabilidade. Este compósito é um material ecológico e de fácil reciclagem, mas se destaca pela sua utilização como isolante térmico e acústico.

Senhoras (2003) destacou as principais características deste compósito:

- Durabilidade: as fibras de coco são constituídas por lignocelulósicos, que influenciam positivamente na durabilidade do material atribuída ao alto teor de lignina, quando comparado a outras fibras naturais.

- Rigidez e impermeabilidade: o elevado índice de rigidez e impermeabilidade apresentado pelo compósito é uma característica presente pela associação da celulose com a lignina.

- Resistência contra-ataques biológicos: a presença de lignina em conjunto com a celulose atua como agente contra-ataques biológicos, apresentando resistência a fungos e roedores.

- Acústico: o material amplia a difusão, podendo ser adotado como material acústico.

- Térmico: a fibra de coco é comumente utilizada como material acústico, por demonstrar baixa condutividade térmica $(0,043$ a $0,045 \mathrm{~W} / \mathrm{mk})$.

\section{Conclusão}

A fibra de coco é um compósito vegetal que possui com alto teor de lignina $(45,0$ - 46,0\%) em sua composição em comparação a outras fibras naturais. Esta composição física faz com que esta fibra apresente características interessantes para diversos setores econômicos, entretanto, a fibra de coco verde possui alto teor de umidade em comparação com a do fruto maduro, fato este que reduz o seu aproveitamento.

\section{Referências}

ALVES, R.M; MULLER, A.A. Aspectos básicos do cultivo do coqueiro (Cocos nucifera L.). Apostila - CPATU/EMBRAPA, Belém - PA, 1995.

ARAGÃO, W.M. et al. Fruto do coqueiro para consumo natural. In: ARAGÃO, W.M. (Ed.). Coco pós-colheita. Brasília: Embrapa, 2002.

BARBOSA, R.A. et al. Extração de fibras de coco para aplicação em materiais de engenharia. ENCONTRO LATINOAMERICANO DE INICIAÇÃO CIENTÍFICA. Universidade do Vale do Paraíba, 2016.

BHATIA, S.K.; SMITH, J.L. Bridging the Gap Between Engineering and the Global Word - A case study of the coconut (coir) fiber industry in Kerala, India, 2008. Synthesis Lectures on Eng. Technol. Soc. Morgan Claypool, v.3, n.1, p.1-58, 2008. doi: 10.2200/S00112ED1V01Y200804ETS006.

BLEDZKI, A.K.; SPERBER, V.E.; FARUK, O. Natural and wood fibre reinforcement in polymers. Reino Unido: Rapra Technology Limited. 2002.

BRAINER, M.S.C.P. Produção de coco: o Nordeste é destaque nacional. Cad. Setorial ETENE, v.3, n.61, 2018.

CABRERA, J.G.; NWAUBANI, S. O. Experimental methods 
for the preparation of palm fruit and other natural fibres for use in reinforced cement composites. In: SOBRAL, H.S. (Ed.). Vegetable Plants and their Fibres as Building Materials. Brazil: RILEM Proceedings of the Second International Symposium, 1990.

MINKE, G. Building with Earth. Design and technology of a Sustainable Architecture. Birkhauser - Publishers for Architecture. Basel, Berlin, Boston. 2012.

PASSOS, P. R. D. A. Destinação sustentável de cascas de coco (cocos nucifera) verde: obtenção de telhas e chapas de partículas. Rio de Janeiro: Universidade Federal do Rio de Janeiro, 2005.

PRITCHARD, M.; SARSBY, R.W.; ARNAND, S.C. Textiles in civil engineering. Part 2 - natural fibre geotextiles. In: HORROCKS, A.R.; ARNAND, S.C. Handbook of technical textiles. Washington: CRC Press, 2000.

RAVEN, P.H.; EVERT, R.F.; EICHHORN, S.E. Biologia vegetal. Rio de Janeiro: Guanabara Koogan, 2014.

ROSA, M.D.F. et al. Caracterização do pó da casca de coco verde usado como substrato agrícola. Fortaleza: Embrapa, 2001.

ROWELL, R.M.; HAN J.S.; ROWELL, J.S. Characterization and factors effecting fiber properties. In: FROLLINI, E.; LEAO, A.L.; MATTOSO, L.H.C. Natural Polymers and Agrofibers Composites. São Carlos: Embrapa, 2000.

SENHORAS, E.M. Estratégias de uma agenda para a cadeia agroindustrial do coco: Transformando a ameaça dos resíduos em oportunidade eco-eficientes. Campinas, SP: Universidade Estadual de Campinas - Instituto de Economia, 2003. 